\title{
MUSCLE FIBER BRANCHING - DIFFERENCE BETWEEN GRAFTS IN OLD AND YOUNG RATS
}

\author{
MICHAEL BLAIVAS ${ }^{a}$ and BRUCE M. CARLSON ${ }^{a, b}$ \\ "Department of Anatomy and Cell Biology and ${ }^{b}$ Institute of Gerontology, University of Michigan, Ann \\ Arbor, Michigan (U.S.A.)
}

(Received March 13th, 1990)

(Revision received January 21st, 1991)

\section{SUMMARY}

Large numbers of branched muscle fibers occur in the freely grafted rat extensor digitorum longus muscle. The ratio of branched/non-branched muscle fibers in grafts is much higher in old ( 24 months) than in young ( 4 months) host rats. Crossage transplants show that the proportion of branched muscle fibers is related to the age of the grafted muscle and not to the age of the host. This is in contrast to mass and maximum isometric tension, in which the age of the host, rather than the age of the grafted muscle, is the determinant of the success of the muscle graft.

Key words: Skeletal muscle; Regeneration; Aging; Muscle fiber branching; Muscle grafts

\section{INTRODUCTION}

A recent study has shown that freely grafted extensor digitorum longus (EDL) muscles regenerate much more poorly in old (24-month) rats than in young (4-month) rats [1]. In the same study, a cross-age transplantation experiment was performed. In highly inbred rats old EDL muscles were grafted in place of the EDL muscles in young rats, and the EDL muscles of the young rats were grafted into the beds of the removed EDL muscles from the old rats. The old-into-young EDL grafts regenerated as well as young-into-young EDL autografts in the contralateral limbs of the same hosts, whereas the young-into-old EDL grafts regenerated no better than

Correspondence to: Dr. Bruce M. Carlson, Department of Anatomy and Cell Biology, 4643 Medical Science Il Bldg., University of Michigan, Ann Arbor, MI 48109-0616, U.S.A. 
old-into-old autografts in the same hosts. This experiment showed that the age of the host, rather than the age of the muscle is the principal determinant of the success of regeneration in the grafts.

Understanding the basis of the age-of-host effect requires a knowledge of the structure of the grafts as well as identification of extrinsic factors that might influence the course of muscle regeneration within the grafts. An obvious structural parameter is the number of muscle fibers in old and young grafts. Do fewer muscle fibers form in muscle grafts in old than in young hosts? In order to make accurate determinations of the number of muscle fibers in the grafts, the technique of nitric acid dissociation was used to separate the individual muscle fibers for counting under a dissecting microscope. This allowed us to avoid a geometric artifact that can render muscle fiber counts from cross-sections inaccurate [2]. During the course of the muscle fiber counting, it became apparent that a high percentage of the regenerated muscle fibers in the grafts was branched and also that there were significant age-related differences in the amount of branching within the grafts. The purpose of the present study was to determine if the high proportion of branched muscle fibers in old muscle autografts was related to the age of the muscle or the age of the host.

\section{MATERIALS AND METHODS}

These experiments were performed on inbred male Fischer-344 rats obtained from the National Institute on Aging. They involved two sets of four control non-grafted EDL muscles from two young (4-month) and two old (24-month) rats and four sets of free muscle grafts ( 3 grafts per set). The types of grafts were as follows: (1) younginto-young (Y-Y) EDL autografts; (2) old-into-old (O-O) EDL autografts; (3) young-into-old (Y-O) cross-age EDL grafts; and (4) old-into-young (O-Y) cross-age EDL grafts. Data from previous experiments [3, unpublished data] have shown no evidence that immunological rejection occurs in the cross-age grafts of either the inbred Wistar or Fischer-344 strains.

Grafting surgery was carried out on pairs of old and young animals anesthetized with methoxyflurane (Metofane, Pitman-Moore, Inc.). In each pair of rats, the left EDL muscle (in both the old and young rats, $\mathrm{O}-\mathrm{O}$ and $\mathrm{Y}-\mathrm{Y}$ ) was completely removed and then autografted in proper orientation back into its own bed. The proximal and distal tendons were sutured to the corresponding tendon stumps. To facilitate reinnervation, the stump of the motor nerve was implanted into the graft. Then the overlying biceps femoris muscle and the skin incision were sutured. In the same pair of rats the right EDL muscle from the old rat was cross-age grafted in place of the right EDL muscle of the young rat (O-Y) and vice versa (Y-O). The surgical technique was otherwise identical to that described for the autografts. After recovery from anesthesia, the rats were placed on oral terramycin for a week. Animal experimentation and care followed the guidelines laid down by the National Research Council. 
Sixty days after surgery, the EDL grafts were removed, placed in a buffered mammalian Ringer solution aerated with $95 \% \mathrm{O}_{2}$ and $5 \% \mathrm{CO}_{2}$ and tested for isometric contractile properties in vitro at $25^{\circ} \mathrm{C}$. The physiological results were reported separately [3] and will not be reiterated here except to say that the cross-age muscle transplantation effect that had been previously reported in inbred Wistar rats [1] was confirmed here on the inbred Fischer 344 rats. After the measurements of contractile properties, the EDL grafts and non-grafted control muscles were removed and frozen in Tissue-Tek (Miles Laboratories) in isopentane cooled by solid $\mathrm{CO}_{2}$. Prior to analysis the muscles were stored in a $-87^{\circ} \mathrm{C}$ freezer.

In preparation for analysis, each muscle was thawed and placed into a solution of $15 \%$ nitric acid for digestion of the connective tissue from the muscle fibers. Digestion times varied with the type of muscle. Control muscles required $8 \mathrm{~h}$, whereas young and old grafts required 24 and up to $35 \mathrm{~h}$, respectively. The digested muscles were then transferred to a solution containing equal parts of glycerol and $0.2 \%$ sodium dodecyl sulfate. Individual muscle fibers were teased apart with fine metal probes, and all muscle fibers were counted and tabulated into specific categories. Differences between group means were evaluated with the Student $t$-test. $P=0.05$ was considered to be the threshold of statistical significance.

Specimens of muscle fibers to be examined by scanning electron microscopy were taken from teased individual muscle fibers or small bundles of muscle fibers. The teased muscle fibers were fixed in a solution of $2.5 \%$ glutaraldehyde $/ 4 \%$ formaldehyde in $0.1 \mathrm{M}$ phosphate buffer. After washing and dehydration in a series graded percentages of ethanol, the muscle fibers were placed in two 10-min changes of hexamethydisilazane (Polysciences) and then placed in a dessicator overnight. After gold sputter-coating, they were examined with an ISI-30 scanning electron microscope.

For transmission electron microscopy, small bundles of muscle fibers were fixed by immersion in $2.5 \%$ glutaraldehyde in $0.1 \mathrm{M}$ phosphate buffer and postfixed in $1 \%$ osmium tetroxide. The tissues were then dehydrated and embedded in Epon. After examination of thick ( $1 \mu \mathrm{m}$ ) sections by light microscopy, thin sections were examined with a Philips 400 transmission electron microscope.

\section{RESULTS}

The total muscle fiber counts revealed no significant differences between young and old control muscles (Fig. 1). Counts of dissociated muscle fibers were significantly reduced in both young and old grafts, but an unexpected finding was the presence of large numbers of branched muscle fibers (Fig. 2) in all groups of grafts, both young and old, and autografts and cross-age grafts (Fig. 1).

Muscle fiber branching followed a large number of patterns, ranging from simple bifurcation at one end of the fiber to very complex aggregates. Scanning and transmission electron microscopy (Figs. 3 and 4) verified the light microscopic analy- 


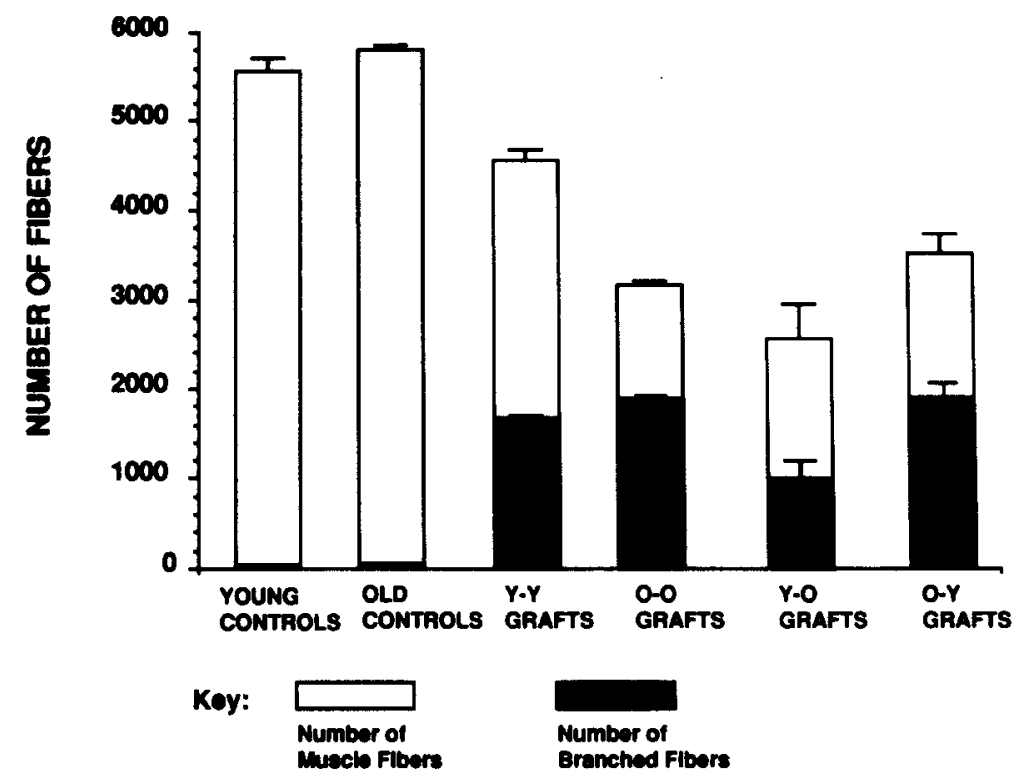

Fig. 1. Mean numbers of total muscle fibers and branched muscle fibers for young and old control muscles and muscle grafts. Young non-grafted controls had a mean of $18 \pm 1.08$ and old controls had a mean of $23.5 \pm 1.19$ branched muscle fibers. The error bars represent standard errors.

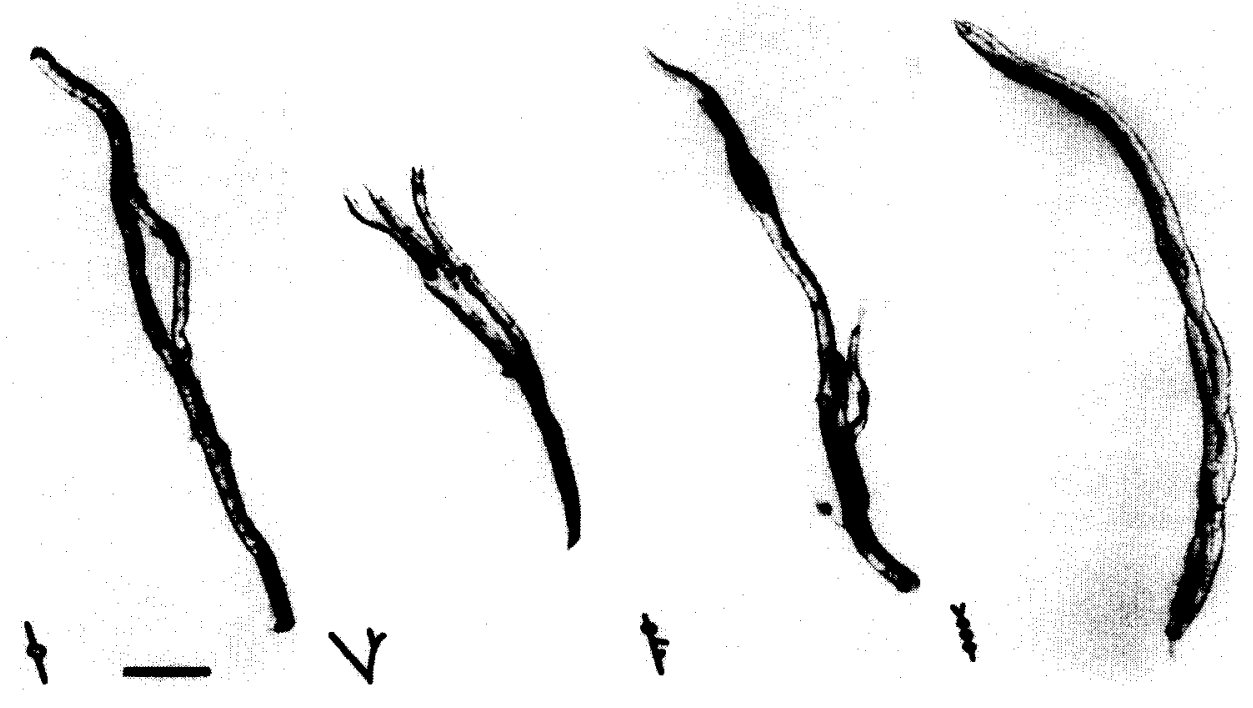

Fig. 2. Examples of typical branching patterns seen in dissociated muscle fibers in grafts. The outlines in the insets show the configuration of these muscle fibers as summarized in Fig. 5. The bar equals $100 \mu \mathrm{m}$. 

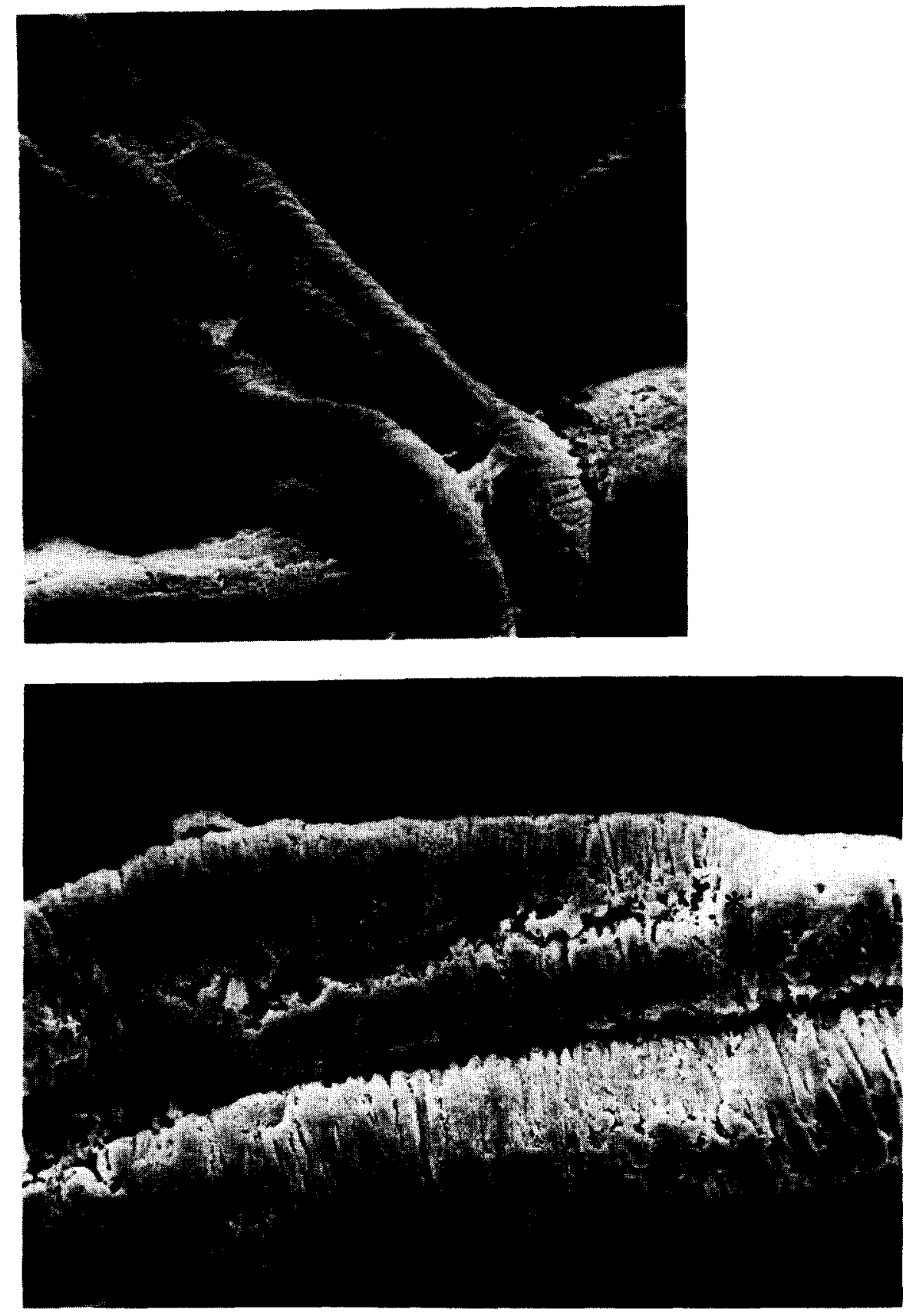

Fig. 3. Scanning electron micrographs of bundles of muscle fibers, illustrating some of the patterns of branching seen in this study. (A) A group of muscle fibers showing two branch points (arrows) and a loop (asterisks on either end of cleft). Bar equals $100 \mu \mathrm{m}$. (B) Higher power magnification of a cleft (asterisks) in a muscle fiber. Bar equals $50 \mu \mathrm{m}$. 
$m$

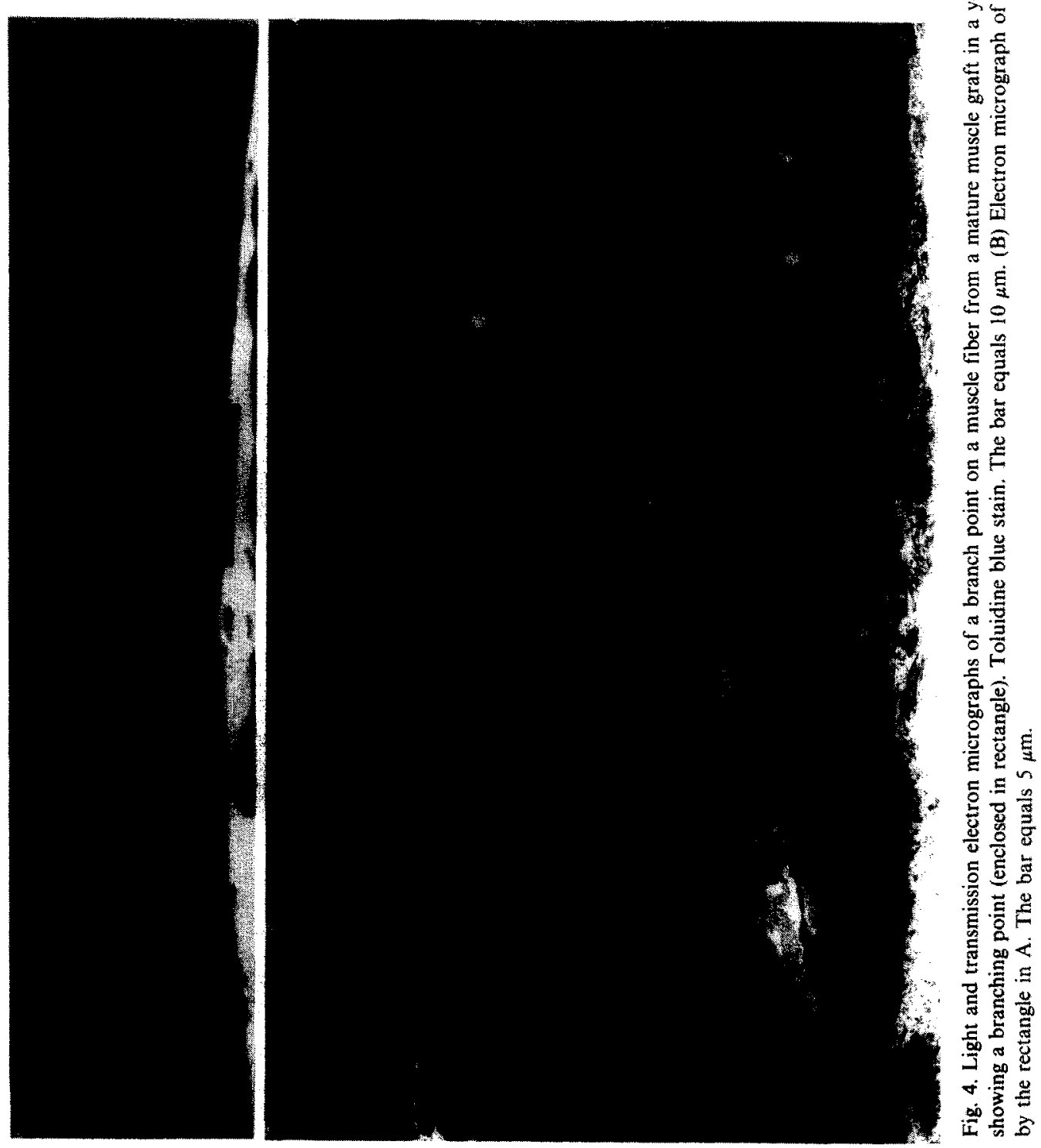




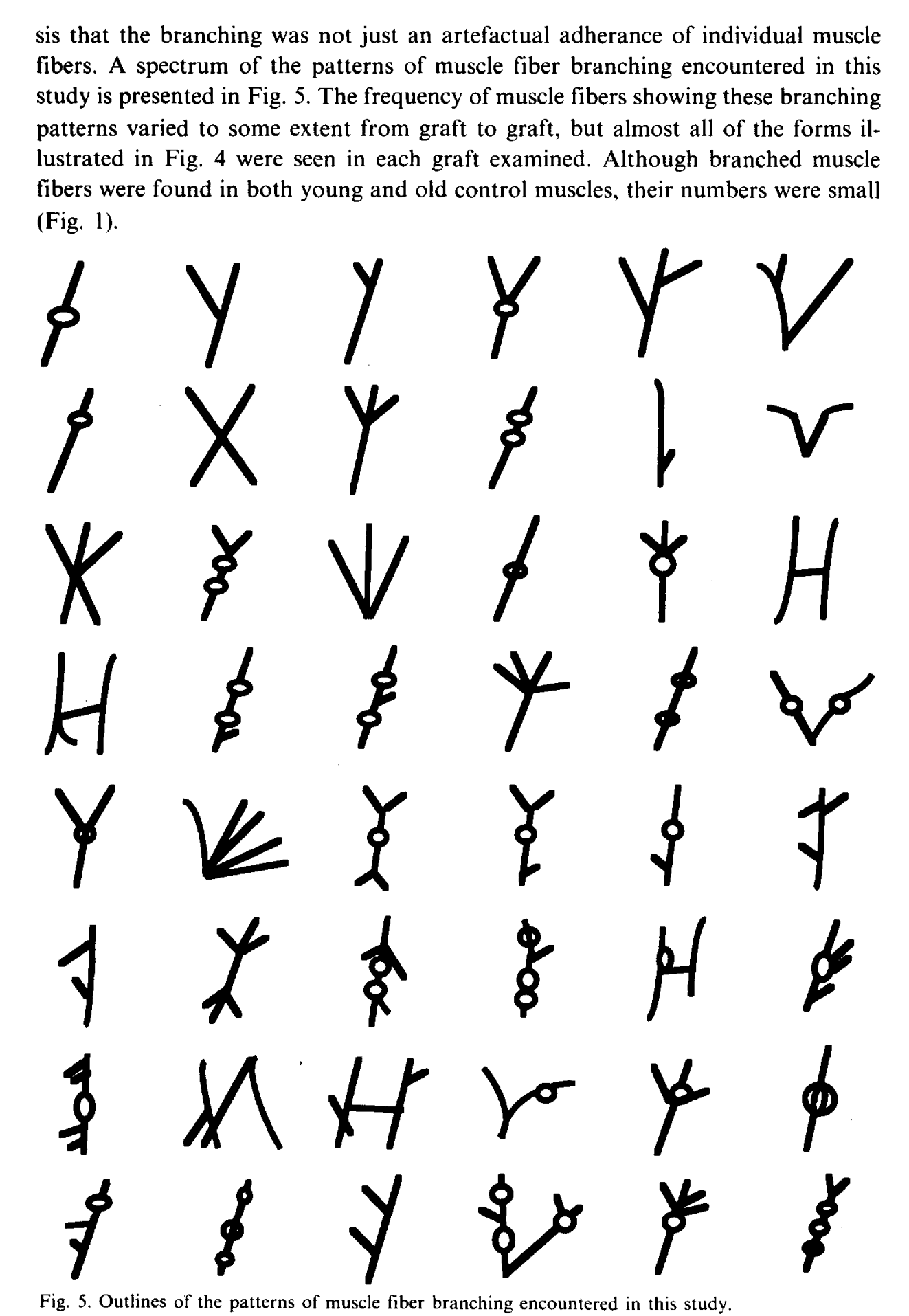


In all types of muscles, both controls and grafts, branched muscle fibers predominated in the middle of the muscle, along both the proximodistal axis and in the cross-sectional plane. There was also a strong tendency for muscle fibers of a given branching configuration to be concentrated in groups. Nevertheless, some branching muscle fibers could be found throughout the muscles.

Particularly in old grafts, large numbers of thin, fragile muscle fibers were found as aggregates, mostly in the distal part of the muscle. In all groups of grafts, as compared with control muscles, the muscle fibers were more tightly adherent to connective tissue, and a longer period of nitric acid digestion was required to tease out the muscle fibers.

The morphological significance of the muscle fiber branching is well illustrated by an examination of the ratios of branched to unbranched muscle fibers (Fig. 6). In the $Y-Y$ autografts the ratio of branched to unbranched muscle fibers was much less $(P<0.005)$ than it was in $\mathrm{O}-\mathrm{O}$ autografts, in which the majority of muscle fibers were branched. The cross-age muscle grafts performed in the contralateral legs of the same animals proved to be instructive. The ratio of branched to unbranched muscle fibers in the $\mathrm{Y}-\mathrm{O}$ grafts did not differ significantly from that in $\mathrm{Y}-\mathrm{Y}$ grafts, and the branching ratio in $\mathrm{O}-\mathrm{Y}$ grafts did not differ significantly from that in O-O grafts.

Although this cross-age grafting model necessarily involved the use of autografts and isografts for contralateral muscles of the same hosts, there was no morphological evidence of immunological incompatibility or of any difference between the autografted and isografted muscles. In addition, comparisons of proportions of

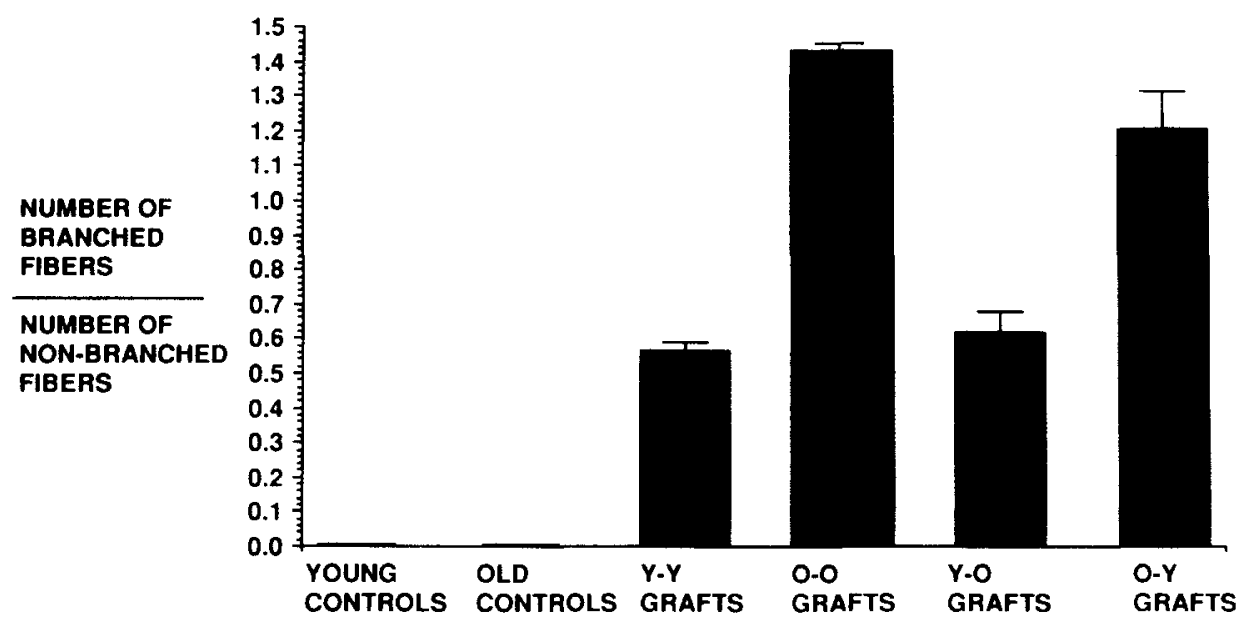

Fig. 6. Ratios of branched to non-branched muscle fibers in control muscles and the various graft combinations used in this study. For young controls the mean ratio is $0.003 \pm 0.0002$, and for old controls it is $0.004 \pm 0.0002$. The error bars represent standard errors. 
branched muscle fibers in muscle grafts taken from the same rats $(0-O$ autografts vs. $\mathrm{O}-\mathrm{Y}$ isografts and $\mathrm{Y}-\mathrm{Y}$ autografts vs. $\mathrm{Y}-\mathrm{O}$ isografts) revealed no significant differences between the autografted and isografted muscles (Table I). Therefore, the only consistent factor to which the proportion of branched muscle fibers could be attributed was the age of the donor muscle.

\section{DISCUSSION}

This study was initially undertaken to determine whether or not there was any correlation between the success of muscle grafts in old and young rats, as measured by mass and isometric tetanic tension, and the number of muscle fibers in these grafts. The data obtained by counting dissociated muscle fibers showed that it is impractical to discuss numbers of muscle fibers when such a high proportion of regenerated fibers is branched. The major problem is whether each dissected branched muscle 'fiber' should be considered a single muscle fiber or whether the individual branches should be factored into the counts.

The high incidence of muscle fiber branching means that it would be very difficult to interpret properly muscle fiber counts made on cross-sections of muscle grafts. In a study of muscle grafts in young rats, Carlson [4] counted muscle fibers in crosssections and found no significant differences between several types of mature grafts and control muscles. In retrospect, it is apparent that many of the muscle fibers counted must have been branched. Interestingly, data on individual muscles from the present study reveal that if all the muscle fiber branches are counted separately, the number of 'muscle fibers' in a given cross-section could equal or even exceed control values.

Unpublished data (Carlson) have shown a significant increase in muscle fibers per cross-section over control values in early ( $2-3$ week) rat EDL grafts. This may be a reflection of a greater incidence of branched muscle fibers in short-term than in mature grafts.

A series of papers from Ontell's laboratory [5-7] reported the presence of branched muscle fibers in both dystrophic and freely grafted muscles of the mouse. They noted that branching appears very early in the post-grafting period, as early as 7 days in the mouse [6]. At this stage, remnants of degenerating muscle fibers are still found in the center of the graft. In a detailed study of the structure of branched muscle fibers, Bourke and Ontell [7] noted two distinct types of branching, one involving a cross-sectional constriction between the parent muscle fiber and the branch and the other involving the accumulation of vesicular membranes at the branch point. In the same study, these authors found that $6.6 \%$ of the muscle fibers branched within $150 \mu \mathrm{m}$ of the plane of the greatest girth of the muscle. Since this distance accounted for about $2 \%$ of the total length of the graft, these authors postulated that considerable numbers of regenerated muscle fibers branch somewhere along their length. For quantitative studies of muscle fiber branching, the dissociation technique 
is an efficient method, but it does not provide the detailed structural information that is obtained in reconstruction studies at the electron microscopic level.

In a study involving reconstruction of muscle fibers from serial light microscopic cross-sections, Schmalbruch [8] reported that muscle biopsies taken from two boys with Duchenne muscular dystrophy contained large numbers of branched muscle fibers. The branching patterns illustrated in his report (e.g. Fig. 1 of Ref. 8) were quite similar to those seen in this study.

Ontell et al. [6] found that the branching of regenerating muscle fibers occurs in both young and old mice, but comparative quantitative data were not obtained. The present study shows that the percentage of branching is clearly increased in muscle fibers regenerating in old rats. With the knowledge of the cross-age transplantation effect, we were interested to see if the amount of muscle fiber branching was a function of the age of the grafts or of the host. The results clearly showed that the incidence of branching is related to the age of the grafted muscle and not to the age of the host (Fig. 5). This is in sharp contrast to muscle mass and contractile functions, which are determined by the age of the host and not the age of the graft [1]. Since in mice, at least, a major type of branching is established very early in regeneration, even before innervation [7], we speculate that the branching pattern in $\mathrm{O}-\mathrm{Y}$ and $\mathrm{Y}-\mathrm{O}$ grafts may be related to some structural property of the grafted muscle.

The mechanism of muscle fiber branching is still little understood. Branching is found in a variety of muscle diseases $[8,9]$ and in hypertrophic muscle after weight lifting [10,1.1] or ablation of synergists [12-16]. In these studies branching has often been interpreted as the result of an overloaded muscle fiber undergoing a longitudinal splitting. Several options remain and these are not mutually exclusive. In addition to splitting of a muscle fiber, branching could result from the secondary fusion of two independent muscle fibers or myotubes. In regeneration, at least, it could also be related to the outward migration of satellite cells through a defect in a basal lamina and their fusion into a branch that is connected with a new muscle fiber regenerating within the basal lamina. It remains to be determined whether the mechanism underlying the appearance of branched muscle fibers is the same in regeneration and hypertrophy.

Up to the time when this study was undertaken all indications suggested that the major characteristics of regenerating muscles in old animals (e.g. lesser mass, poorer histological structure and reduced maximum tetanic force) were reversible when the old muscles were grafted into young hosts [1]. In other words, the environment provided by the young host, rather than the chronological age of the grafted muscle, determined the overall course of muscle regeneration. A recent report by Gulati [17], involving the grafting of muscles between diabetic and normal rats, reinforced this view of the powerful role of the environment as a determinant of the success of muscle regeneration. He found that diabetic muscles became normalized when grafted into normal hosts and that normal muscles grafted into diabetic hosts took on the properties of diabetic muscles. 
The data from this study on numbers of branched muscle fibers demonstrate one property of regenerating old muscle that is retained when the muscles are grafted into young hosts. Since the mechanism of muscle fiber branching is still not clearly understood in any system, including the muscle grafting model, we can do no more than document the phenomenon at this time. Nevertheless in this restricted system, at least, the proportion of branched muscle fibers could be considered a stable 'biomarker of aging' that could prove useful in further studies.

\section{ACKNOWLEDGEMENTS}

This study was supported by NIH grant DE-07687. The authors thank Tom Komorowski for expert technical assistance.

\section{REFERENCES}

1 B.M. Carlson and J.A. Faulkner, Muscle transplantation between young and old rats: age of host determines recovery. Am. J. Physiol, 256 (1989) C1262-C1266.

2 L.C. Maxwell, J.A. Faulkner and G.J. Hyatt, Estimation of the number of fibers in guinea pig skeletal muscle. J. Appl. Physiol., 37 (1974) 259-264,.

3 B.M. Carlson, J.A. Faulkner, Skeletal muscle regeneration and aging, In Karcher, H. (ed): Functional Surgery of the Head and Neck, RM-Druck and Verlagsgesellschaft, Graz, Austria, 1989, pp. $131-134$.

4 B.M. Carlson, A quantitative study of muscle fiber survival and regeneration in normal, predenervated, and Marcaine-treated free muscle grafts in the rat. Exp. Neurol., 52 (1976) $421-432$.

5 M. Ontell and K.C. Feng, The three-dimensional cytoarchitecture and pattern of motor innervation of branched striated myotubes. Anat. Rec., 200 (1981) $11-31$.

6 M. Ontell, D. Hughes and D. Bourke, Secondary myogenesis of normal muscle produces abnormal myotubes. Anat. Rec., 204 (1982) 199-207.

7 D. Bourke and $M$. Ontell, Branched myofibrils in long-term whole muscle transplants: A quantitative study. Anat. Rec., 209 (1984) $281-288$.

8 H. Schmalbruch, Regenerated muscle fibers in Duchenne muscular dystrophy: A serial section study. Neurology, 34 (1984) 60-65.

9 W.G. Bradley, Muscle fiber splitting, In A. Mauro (ed.), Muscle Regeneration. Raven Press, New York, 1979, pp. 215-232.

10 W. Gonyea, G.C. Ericson and F. Bonde-Petersen, Skeletal muscle fiber splitting induced by weightlifting exercise in cats. Acta Physiol. Scand. 99 (1977) $105-109$.

11 K.W. Ho, R.R. Roy, C.D. Tweedle, W.W. Heusner, W.D. Van Huss and R.E. Carrow, Skeletal muscle fiber splitting with weight lifting exercise in rats. B. J. Anat., 157 (1980) 433-440.

12 M.H. Snow and B.S. Chortkoff, Frequency of bifurcated muscle fibers in hypertrophic rat soleus muscle. Muscle and Nerve, 10 (1987) 312-317.

13 E.C.B. Hall-Craggs, The longitudinal division of fibres in overloaded rat skeletal muscle. J. Anat.. 107 (1970) $459-470$.

14 C.D. Ianuzzo, P.D. Gollnick and R.B. Armstrong, Compensatory adaptations of skeletal muscle fiber types to a long-term functional overload. Life $S c i ., 19$ (1976) 1517-1524.

15 R.W.D. Rowe and G. Goldspink, Surgically induced hypertrophy in skeletal muscles of the laboratory mouse. Anat. Rec., 16I (1968) 69-76.

16 H.S. Vaughan and G. Goldspink, Fibre number and fibre size in a surgically overloaded muscle. $J$. Anat., 129 (1979) 293-303.

17 A.K. Gulati, A comparison of skeletal muscle regeneration in normal and streptozotocin induced diabetic rats. Anat. Rec., 226(1990) 39A. 